\title{
The Performance Characterization of Discrete Kalman Filter under the First Order Autoregressive Demands
}

\author{
Karin Kandananond* \\ Valaya Alongkorn Rajabhat University, Klong-Luang District, Prathumthani Province, Thailand 13180
}

*Corresponding Author: kandananond@ hotmail.com

\begin{abstract}
The accuracy of forecasting is important to the performance of supply chain systems. This research focuses on the demand which is stationary. Since Box-Jenkin's first order autoregressive model or AR (1) is widely used to characterize the stationarity, the data in this research is simulated on the basis of the AR (1) model. Moreover, because Kalman filter is a recursive technique used to forecast the observation, the objective is to facilitate the utilization of Kalman filter by practitioners. The empirical study is conducted to characterize the performance of Kalman filter when the structure of observations follows AR (1) model. Due to the experimental results, the different degrees of stationarity are adjusted by controlling the values of autoregressive coefficients ( $\phi$ ). Afterwards, Kalman filter is applied to forecast the simulated data while the forecasting errors in term of minimum average percentage error (MAPE) are calculated. The results indicate that Kalman filter might work at its best when the autoregressive coefficient is highly positive. After the assessment, the application of Kalman filter to AR (1) observations is completely standardized so it provides the procedures and guidelines for practitioners when dealing with the stationary process.
\end{abstract}

Keywords: Autoregressive, Demand, Forecasting, Kalman Filter, Stationary.

\section{Introduction}

The precision of demand forecasting is important to the performance of supply chain systems. There are many forecasting methods used for demand prediction. A criterion to select the appropriate technique depends on the stationarity of data, stationary and non-stationary. Box Jenkin's autoregressive integrated moving average (ARIMA) is widely used to explain the stationarity structure of data. A subclass of ARIMA, first order autoregressive (AR (1)) model, is utilized to explain stationary observations. However, since there are noises or disturbances embedded in the process, it is hard to apply a specific forecasting technique which is able to handle all the fluctuation in the observations. As a result, Kalman filter, a recursive estimating technique commonly used in the digital signal processing area, is introduced for the estimation because of its advantage, the adaptive capability. Elaborately, a correction step is added to the existing estimates to improve the prediction result. Moreover, there are many applications for Kalman filter in different disciplines, e.g., forecasting power demand and economically related demands. Examples are shown in the works of Inglesi-Lotz ${ }^{(1)}$ and Arisoy and Ozturk ${ }^{(2)}$. They utilized Kalman filter to improve the estimation of the future electricity demand. Moreover, Kalman filter is also applied to improve the demand estimation for supply chain systems. Gursoy and Erkip $^{(3)}$ reported that Kalman filter was also used effectively for multivariate forecasting when demand was correlated. The market prices of products were also smoothed and forecasted when Kalman filter was applied to the selling price. Therefore, Kalman filter was used for the whole process of supply chain including procurement, inventory and production. Moreover, Sun and Deng $^{(4)}$ used Kalman technique to forecast the oil demand in China while the same method was deployed to estimate the water demand by Nasseri, Moeini and Tabesh ${ }^{(5)}$. 
Chaolong et al. ${ }^{(6)}$ had utilized Kalman filter method to predict short-term changing trend and irregularity occurred in time series. For financial application, Huang et al. ${ }^{(7)}$ had integrated Kalman filter with support vector machine method to forecast the option price in Taiwan future exchange while $\mathrm{Vo}^{(8)}$ applied Kalman filter to predict the interest rate with risk-free condition and with risk premium. Kalman filter was also extensively used to the environmental area, e.g., forecasting air quality (Ridder et al. ${ }^{(9)}$ ) and wind speed (Cassola and Burlando ${ }^{(10)}$ ),

Therefore, Kalman filter is successively applied to improve the forecasting capability but the research framework is limited for the case-by-case basis only. There are only few works applying Kalman filter to the standardized stationary model, i.e., AR (1). Brouste and Kleptsyna $^{(11)}$ had analyzed the performance of Kalman filter when the observations are d-dimensional autoregressive noises which were simulated by following the pattern of the first order autoregressive, AR (1), and the first order moving average, MA (1), models. Wang ${ }^{(12)}$ had developed $\mathrm{R}$ package to fit continuous autoregressive model of order $p$ with unequally sampled time series. The coefficients of AR model were calculated by using Kalman filter algorithm. Ferreira, Rodriguez and Lagos ${ }^{(13)}$ deployed Kalman filter and Monte Carlo simulation to estimate the parameters of a non-stationary model. According to the literature, since there are some limitations regarding the performance assessment of Kalman filter, it is interesting to characterize the capability of Kalman filter under the stationary scenario which is based on the AR(1) model. The empirical study is conducted by adjusting the value of AR parameters and Kalman filter is implemented to forecast the simulated observations. Hence, there are standardized guidelines for implementing this technique when practitioners have to deal with the demand forecasting.

\section{Methodology}

Kalman filter is a mathematical algorithm developed by R. E. Kalman in 1960s and it is widely used in time series analysis. The basic concept of Kalman filter is built upon observations measured over time. Since these measurements contain random noises, they are utilized to predict the value of unknown variable. Moreover, the result is proved to be more accurate than the one from a single measurement. Kalman filter is mainly based on the following two models, process or plant model and measurement model in (1) and (2),

$$
\begin{aligned}
& x_{k}=A x_{k-1}+w_{k} \\
& z_{k}=x_{k}+v_{k}
\end{aligned}
$$

, where $x_{k}$ and $x_{k-1}$ are observations at time $\mathrm{k}$ and $\mathrm{k}-1$,

A is a transition matrix,

$w_{k}$ and $v_{k}$ are process noise and measurement noise.

Afterwards, a recursive process is utilized on the estimation of noise parameters (mean and standard deviation of noise). This process is based on two stages, time update (prediction) and measurement update (correction), at a time. Time update equations are shown in (3) as follows:

$$
\hat{x}_{k}^{-}=A \hat{x}_{k-1}^{-}+B u_{k}
$$

The purpose of (3) is to project the value of the forecast for the state ahead $(\mathrm{k})$ along with (4) which is used to project the error covariance,

$$
P_{k}^{-}=A P_{k-1} A^{T}+Q
$$

, where $\mathrm{Q}$ is the process noise covariance.

Next, Kalman gain for the state ahead $\left(\mathrm{K}_{\mathrm{k}}\right)$ is computed as a multiplicative factor to assist in improving the predicted value as shown in (5).

$$
K_{k}=P_{k}^{-} H^{T}\left(H P_{k}^{-} H^{T}+R\right)^{-1}
$$

, where $\mathrm{R}$ is the measurement noise covariance.

When the actual value at the next state $\left(x_{k}\right)$ is observed, it will be utilized to update the measurement as shown in (6).

$$
\hat{x}_{k}=\hat{x}_{k}^{-}+K_{k}\left(z_{k}-H \hat{x}_{k}^{-}\right)
$$

Besides the measurement, the error covariance at time $\mathrm{k}\left(\mathrm{P}_{\mathrm{k}}\right)$ is also updated in $(7)$.

$$
P_{k}=\left(I-K_{k} H\right) P_{k}^{-}
$$

The model widely used to represent stationary processes is the first order autoregressive, AR (1) or ARIMA (1, 0, 0), which is a subclass of Box-Jenkin's autoregressive integrated moving average (ARIMA) model. The equation of AR (1) is shown in (8),

$$
x_{k}=\phi x_{k-1}+\varepsilon_{k}
$$

, where $\phi$ is autoregressive coefficient, $\varepsilon_{k}$ is white noise at time $\mathrm{k}$.

According to (8), AR (1) is a linear model with white noise and has the same pattern as (1) where $\mathrm{A}=\phi$ and $w_{k}=$ $\varepsilon_{k}$. Therefore, AR (1) model is utilized to simulate demand processes in this study. Based on AR (1) process, Kalman filter is applied to compute the following equations as shown in (9) and (10).

$$
\begin{aligned}
& \hat{x}_{k}^{-}=\phi \hat{x}_{k-1}^{-}+B u_{k} \\
& P_{k}^{-}=\phi^{2} P_{k-1}+Q
\end{aligned}
$$

Substitute $P_{k}^{-}$into (5), then 


$$
K_{k}=\frac{\phi^{2} P_{k-1} H}{H^{2} P_{k}+R}
$$

Therefore, the updated forecasting value is equal to

$\hat{x}_{k}=\phi \hat{x}_{k-1}^{-}+B u_{k}+\frac{\phi^{2} P_{k-1} H}{H^{2} P_{k}+R}\left\{z_{k}-H\left(\phi \hat{x}_{k-1}^{-}+B u_{k}\right)\right\}(12)$

and the updated error covariance is

$$
P_{k}=\left(1-\frac{\phi^{2} P_{k-1} H^{2}}{H^{2} P_{k}+R}\right)\left(\phi^{2} P_{k-1}+Q\right)
$$

The estimation process of Kalman filter is shown in Fig. 1 which illustrates two important steps of the recursive, correction (measurement update) and prediction (time update), respectively. When $i$ is equal to $\mathrm{k}-1$, the observation at a period behind $(\mathrm{k}-2)$ is used to derive the estimation at $\mathrm{i}=\mathrm{k}-1\left(\hat{x}_{k-1}^{-}\right)$. Afterwards, the forecast is updated by the measurement update process, the corrected estimation is improved to be $\hat{x}_{k-1}$.

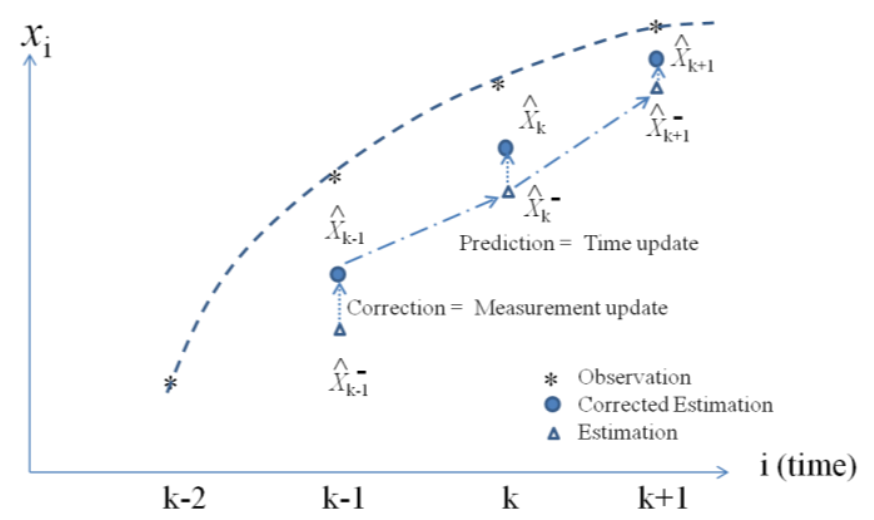

Fig.1. Kalman Filter Process.

After Kalman filter is applied to the observations, the performance index of the proposed method is the forecast errors, difference between the actual and estimation values. The measurement index of the forecast accuracy is represented in term of mean absolute percentage error (MAPE), $\frac{1}{n} \sum_{i=1}^{n} \frac{\left|x_{i}-\hat{x}_{i}\right|}{x_{i}}$.

\section{Simulation Study}

The empirical study was conducted to assess the performance of Kalman filter algorithm utilized to forecast the stationary processes which are based on the AR (1) model. For each value of AR coefficient $(\phi)$, a number of 10,000 iterations of AR (1) processes are simulated while the average forecasting errors are calculated to indicate the performance of Kalman filter when the observations are correlated with the pattern of AR (1) model. The process was simulated at $\phi=0.1,0.2,0.3,0.4,0.5,0.6,0.9$ while the length of simulation period was 80 weeks. For the simulation, the initial conditions of input parameters were set as follows: $\mathrm{A}=\phi \quad \mathrm{H}=1 \mathrm{~B}=0 \mathrm{Q}=10 \mathrm{R}=10 \quad w_{k}=$ 10 and $50 v_{k}=0$. To execute the simulation, M-file code was programmed and run on Matlab. After the simulation, the average values of MAPEs were calculated so the performance of Kalman filter at each degree of $\phi$ was asessed.

\section{Empirical Results}

After the stationary data of AR (1) pattern was simulated, Kalman filter was applied to forecast the observations. Numerically, the forecast errors in term of average MAPE for the different values of $\phi$ were computed and shown in Table 1.

Table 1. Average MAPE.

\begin{tabular}{|c|c|c|}
\hline \multirow{2}{*}{$\boldsymbol{\phi}$} & \multicolumn{2}{|c|}{ Average MAPE } \\
\cline { 2 - 3 } & $\boldsymbol{w}_{\boldsymbol{k}}=\mathbf{1 0}$ & $\boldsymbol{w}_{\boldsymbol{k}}=\mathbf{5 0}$ \\
\hline 0.1 & $5.01 \%$ & $5.12 \%$ \\
\hline 0.2 & $4.54 \%$ & $4.86 \%$ \\
\hline 0.3 & $4.18 \%$ & $4.41 \%$ \\
\hline 0.4 & $4.02 \%$ & $4.13 \%$ \\
\hline 0.5 & $3.82 \%$ & $3.77 \%$ \\
\hline 0.6 & $3.48 \%$ & $3.52 \%$ \\
\hline 0.7 & $3.19 \%$ & $3.18 \%$ \\
\hline 0.8 & $2.84 \%$ & $2.99 \%$ \\
\hline 0.9 & $2.33 \%$ & $2.74 \%$ \\
\hline
\end{tabular}

Due to Table 1 , when $w_{k}$ is set at 10 , the performance of Kalman filter is getting better when the value of $\phi$ is highly positive. At $\phi=0.1$, the forecasting error is as high as $5.01 \%$. However, when $\phi$ increases to 0.2 , Kalman filter seems to have a better performance because the average MAPE gradually drops to $4.54 \%$. Finally, the error decreases to $2.33 \%$ when $\phi$ equals to 0.9 . For $w_{k}=50$, it leads to the similar results. Therefore, the study indicates that the average MAPE gradually decreases when $\phi$ gets increasing. On the other hand, the relationship between errors and $\phi$ is inversely proportional. To demonstrate the performance of Kalman filter graphically, the observations and forecasts at each $\phi$ (when $w_{k}=10$ ) are randomly 
selected and shown in Fig. 2-10.

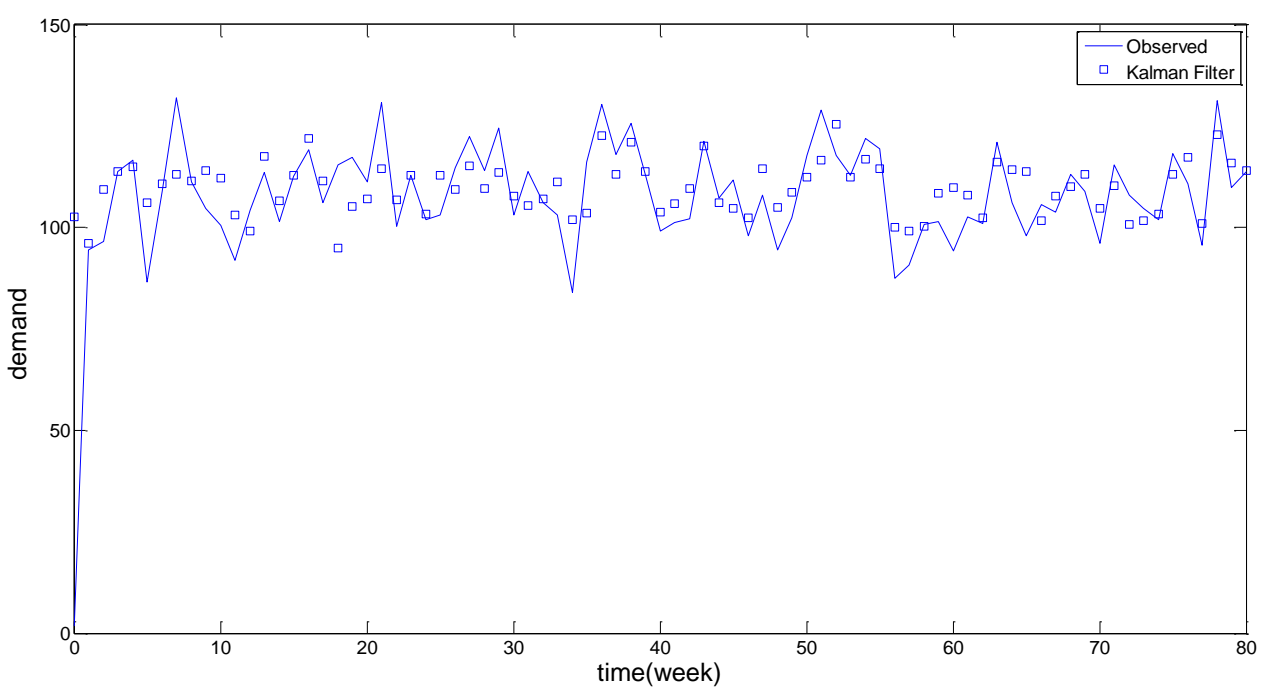

Fig. 2. Observation and Forecasts at $\phi=0.1$ and $w_{k}=10$.

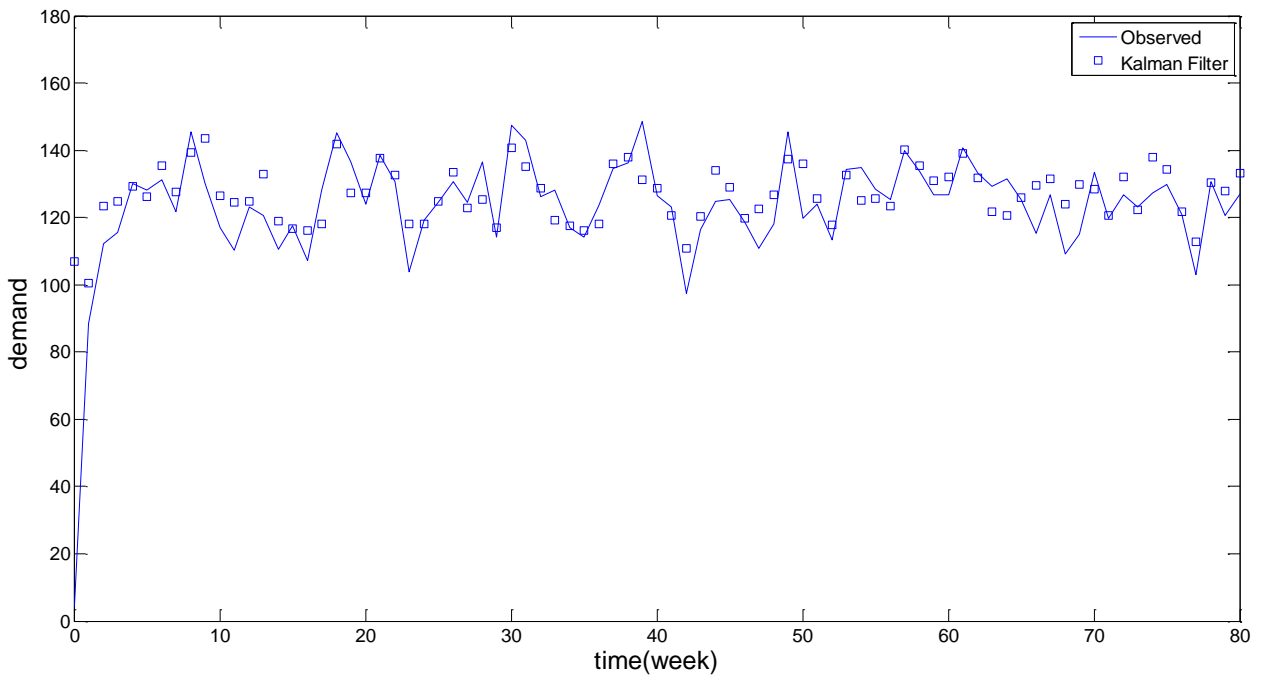

Fig. 3. Observation and Forecasts at $\phi=0.2$ and $w_{k}=10$.

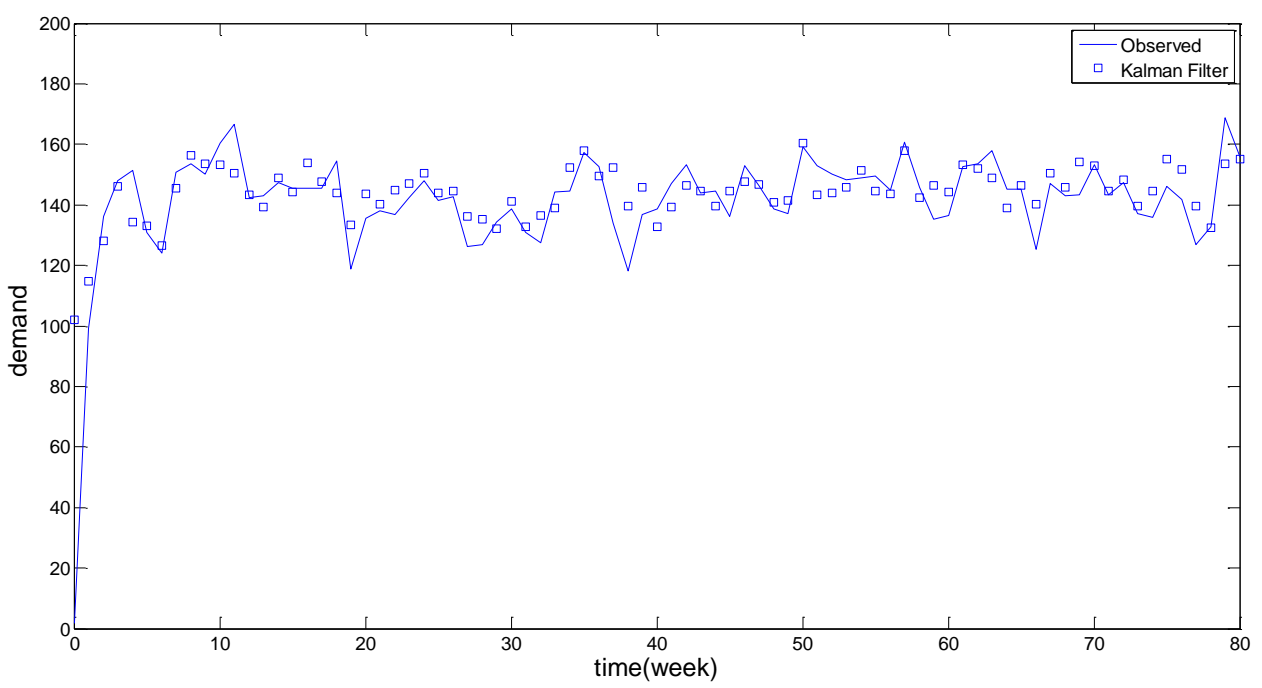

Fig. 4. Observation and Forecasts at $\phi=0.3$ and $w_{k}=10$. 


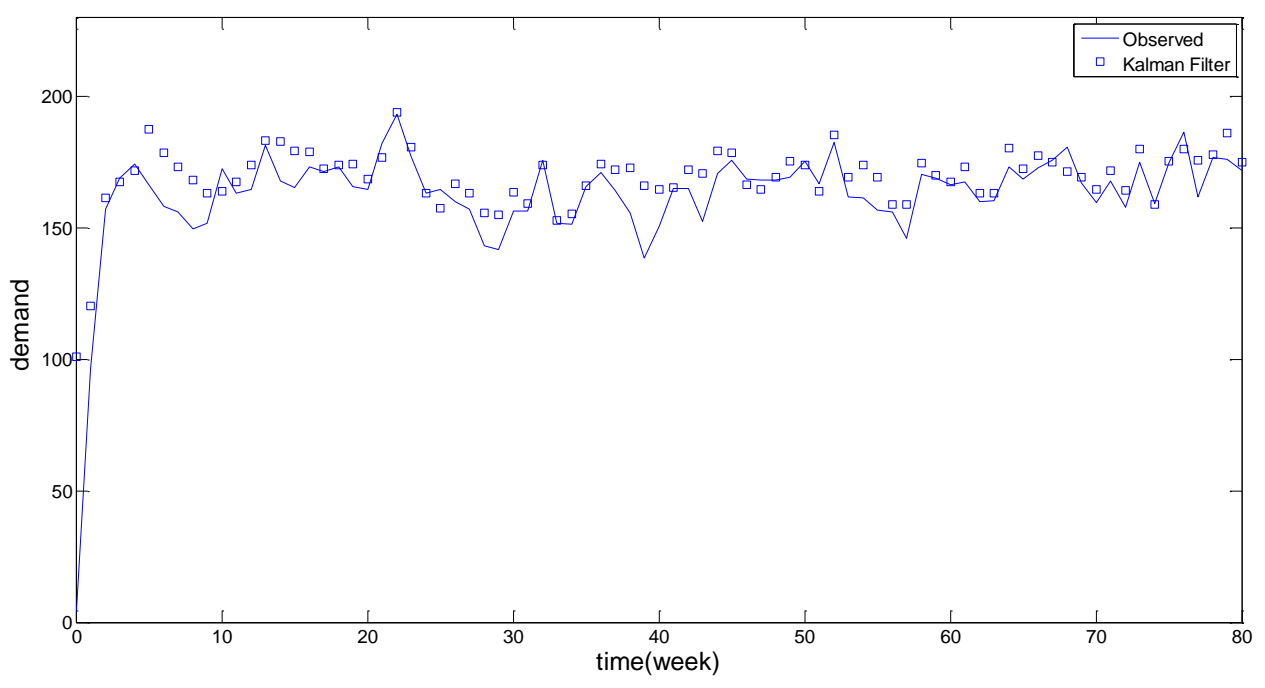

Fig. 5. Observation and Forecasts at $\phi=0.4$ and $w_{k}=10$.

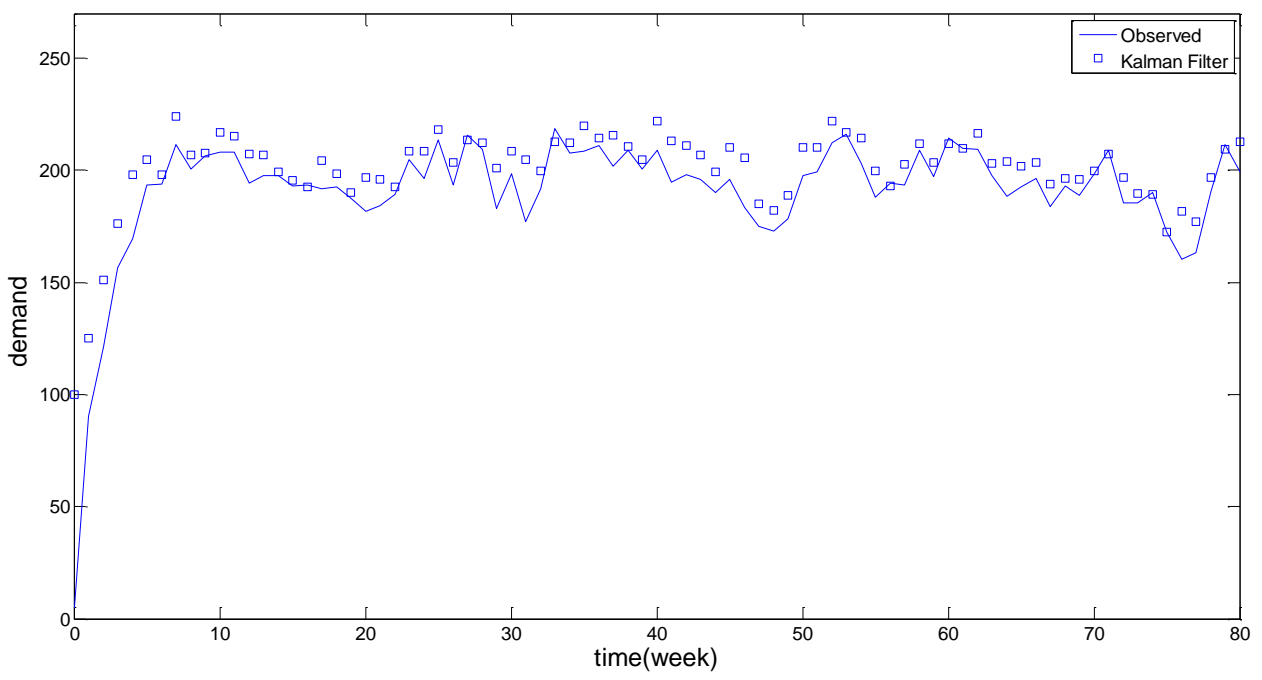

Fig. 6. Observation and Forecasts at $\phi=0.5$ and $w_{k}=10$.

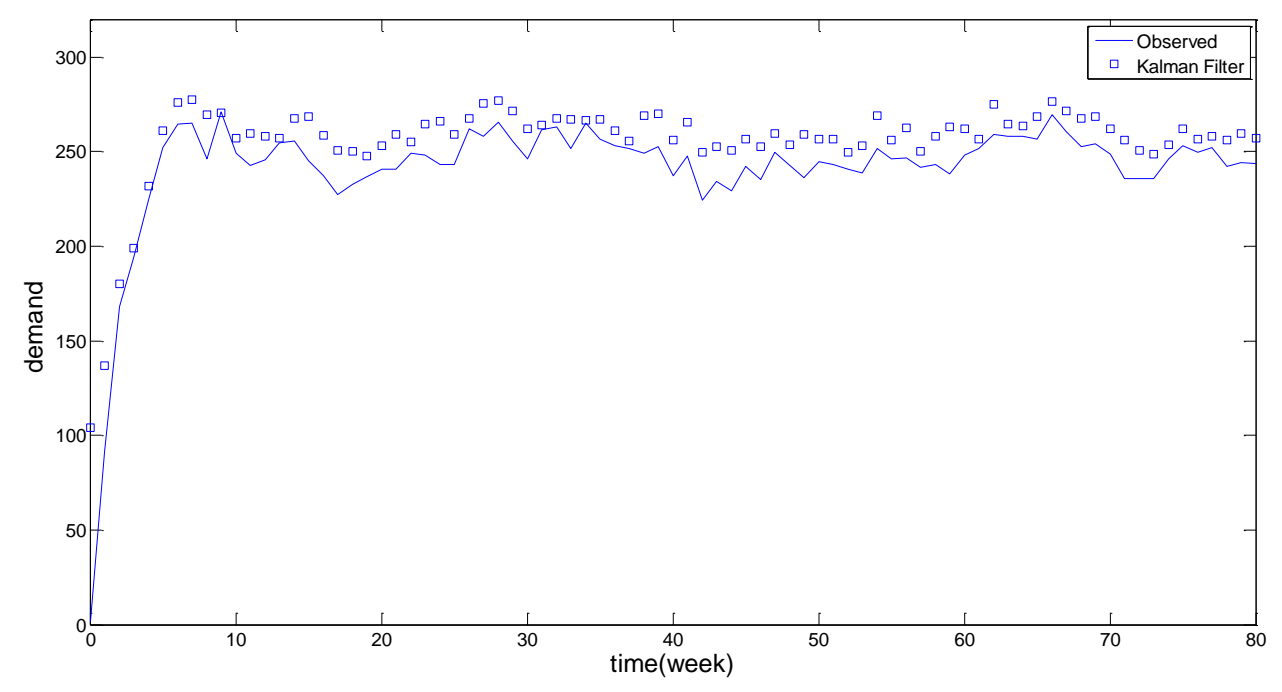

Fig. 7. Observation and Forecasts at $\phi=0.6$ and $w_{k}=10$. 


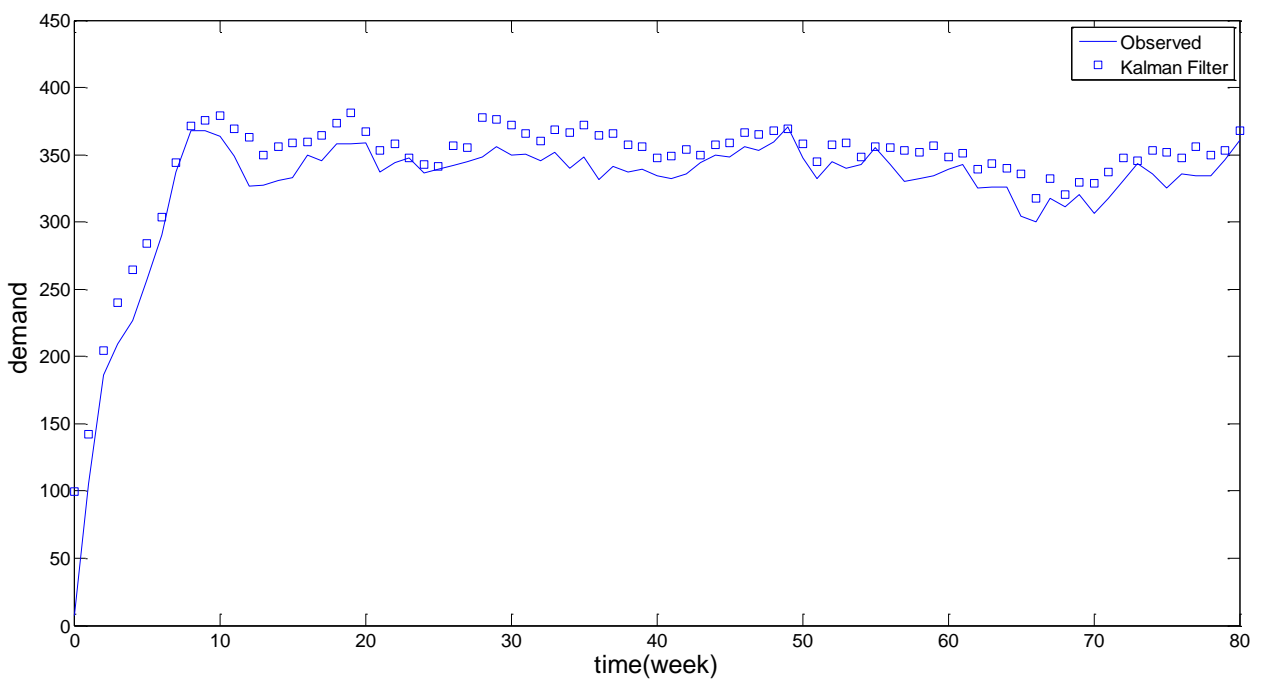

Fig. 8. Observation and Forecasts at $\phi=0.7$ and $w_{k}=10$.

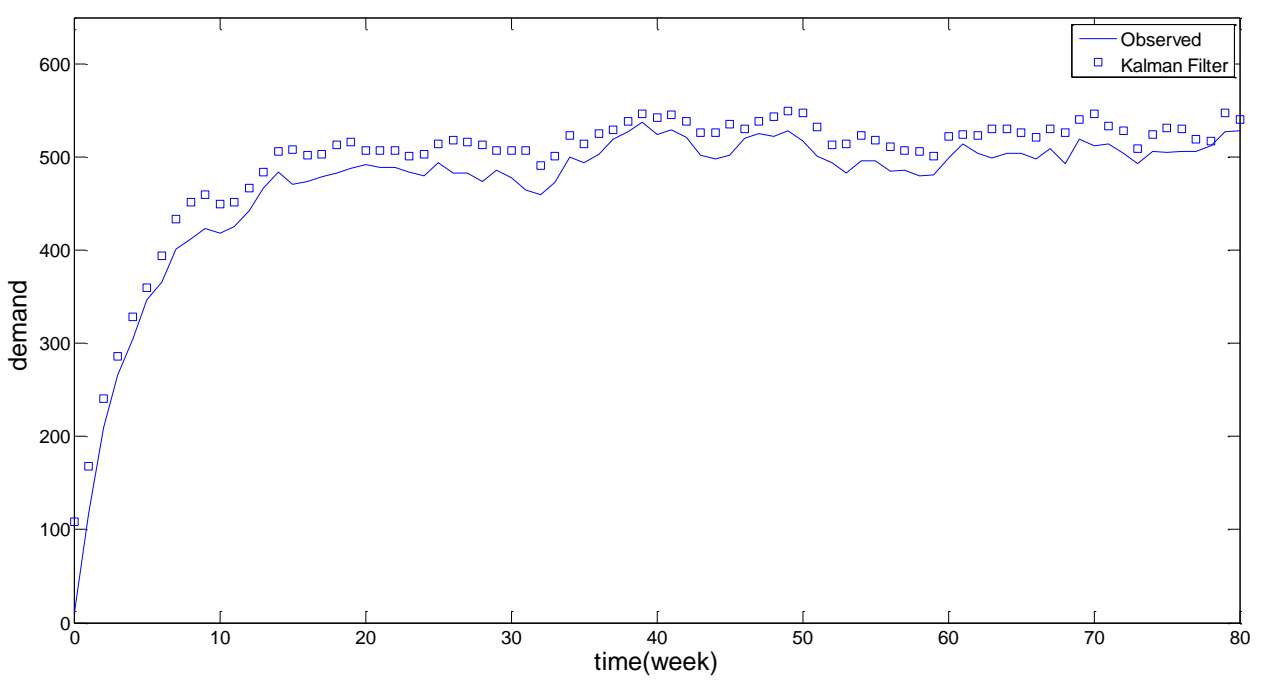

Fig. 9. Observation and Forecasts at $\phi=0.8$ and $w_{k}=10$.

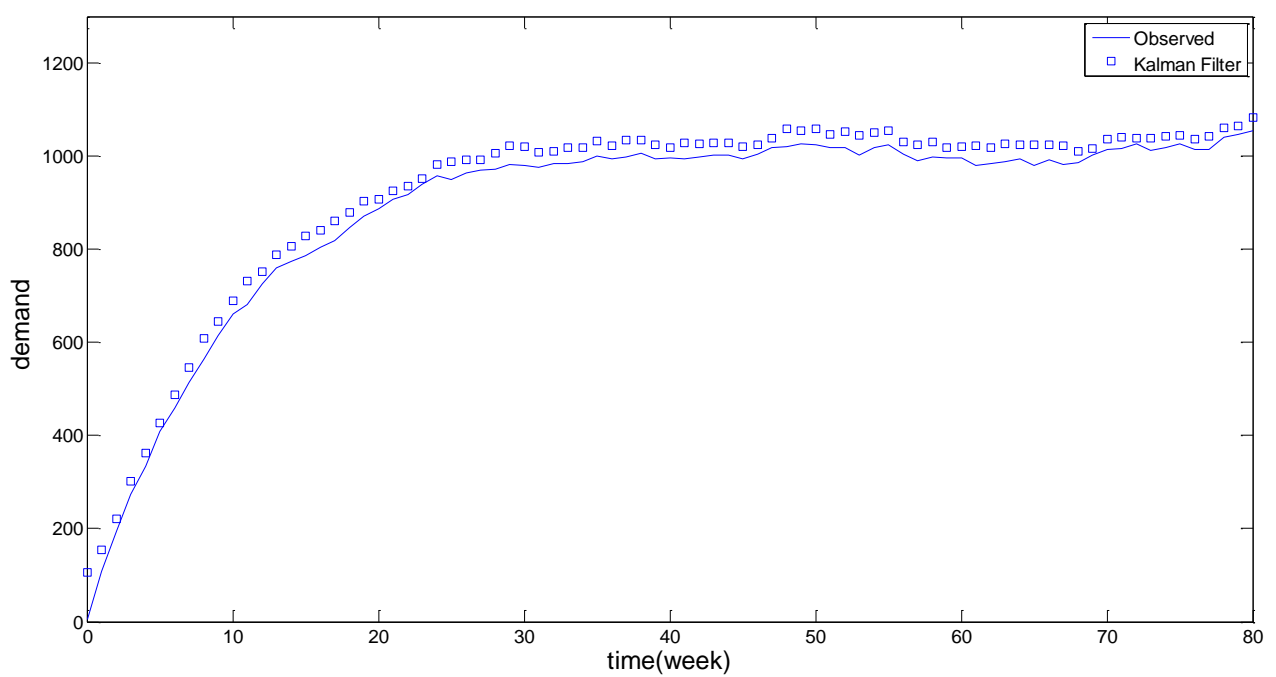

Fig. 10. Observation and Forecasts at $\phi=0.9$ and $w_{k}=10$. 


\section{Conclusions}

Kalman filter is another estimating technique which has the capability to predict the correlated demand. This research focuses on the observations following AR (1) model which is stationary. To assess the performance of Kalman filter, the forecasting errors (MAPE) are calculated after Kalman filter was employed to forecast the simulated observations. The stationary processes are simulated by varying the value of autocorrelation coefficients from $\phi=$ 0.1 to 0.9 . The number of iterations for each $\phi$ are 10,000 . The empirical results show that Kalman filter works effectively when $\phi$ is highly positive since the average MAPE gradually decreases when $\phi$ increases.

\section{Discussions}

According to the results, they explicitly show that Kalman filter has the potential to the forecast the autocorrelation structure of the observations because the average MAPEs are low, i.e., the forecasting errors are ranged from 2.33 to 5.01 percent $\left(w_{\mathrm{k}}=10\right)$ and from 2.74 to 5.12 percent $\left(w_{\mathrm{k}}=50\right)$. Obviously, these percentage errors are not significantly different at $w_{\mathrm{k}}=10$ and 50. Therefore, it implies that the Kalman filter is robust to the process noise or the deviation of the demand in the system. Therefore, it is an appropriate method to forecast the stationary demand.

\section{References}

(1) Roula Inglesi-Lotz : "The Evolution of Price Elasticity of Electricity Demand in South Africa: A Kalman Filter Application", Energy Policy, Vol. 39, No. 6, pp. 3609-3696, 2011.

(2) Ibrahim Arisoy, and Ilhan Ozturk: "Estimating Industrial and Residential Electricity Demand in Turkey: A Time Varying Parameter Approach", Energy, Vol. 66, No. 1, pp. 959-964, 2014.

(3) Melike Baykal-Gürsoy, and Nesim K. Erkip: "Forecasting for Inventory Planning under Correlated Demand", Wiley Encyclopedia of Operations Research and Management Science, Vol. 1, pp. 1-7, 2010.

(4) Mei Sun, and Xing Deng: "Application of Time Series and Kalman Filter Estimation in Oil Demand", International Journal of Nonlinear Science, Vol. 15, No. 3, pp. 284-288, 2013.
(5) Mohsen Nasseri, Ali Moeini, and Massoud Tabesh: "Forecasting Monthly Urban Water Demand using Extended Kalman Filter and Genetic Programming", Expert Systems with Applications, Vol. 38, No. 6, pp. 7387-7395, 2011.

(6) Jia Chaolong, Xu Weixiang, Wang Futian, and Wang Hanning: "Track Irregularity Time Series Analysis and Trend Forecasting", Discrete Dynamics in Nature and Society, Vol. 2012, pp. 1-15.

(7) Shian-Chang Huang, Nan-Yu Wang, Tzu-Ying Li, Yi-Ching Lee, Lung-Fu Chang, and Tzu-Hui Pan: "Financial Forecasting by Modified Kalman Filters and Kernel Machines", Journal of Statistics and Management Systems, Vol. 16, No. 2-03, 2013.

(8) Long H. Vo: "Application of Kalman Filter on Modelling Interest Rates", Journal of Management Science, Vol. 1, No. 1, pp. 1-15, 2014.

(9) Koen De Ridder, , Ujjwal Kumar, Dirk Lauwaet, Lisa Blyth, and Wouter Lefebvre: "Kalman Filter-based Air Quality Forecast Adjustment”, Atmospheric Environment, Vol. 50, pp. 381-384, 2012.

(10) Federico Cassola, and Massimiliano Burlando: "Wind Speed and Wind Energy Forecast through Kalman Filtering of Numerical Weather Prediction Model Output”, Applied Energy, Vol. 99, pp. 154-166, 2012.

(11) Alexandre Brouste, and Marina Kleptsyna: "Kalman Type Filter under Stationary Noises", Systems \& Control Letters, Vol. 61, No. 12, pp. 1229-1234, 2012.

(12)Zhu Wang: "An R Package for Continuous Time Autoregressive Models via Kalman Filter", Journal of Statistical Software, pp. 1-20, 2013.

(13) Guillermo Ferreira, Alejandro Rodríguez, and Bernardo Lagos: "Kalman Filter Estimation for a Regression Model with Locally Stationary Errors", Computational Statistics \& Data Analysis, Vol. 62, pp. 52-69, 2013. 